\title{
Aggrecan Is Degraded by Matrix Metalloproteinases in Human Arthritis Evidence That Matrix Metalloproteinase and Aggrecanase Activities Can Be Independent
}

\author{
Amanda J. Fosang, ${ }^{\star}$ Karena Last, ${ }^{\star}$ and Rose A. Maciewicz ${ }^{\ddagger}$ \\ *University of Melbourne, Department of Paediatrics, Orthopaedic Molecular Biology Research Unit, Royal Children's Hospital, \\ Parkville, 3052, Australia; and ${ }^{\ddagger}$ Cardiovascular and Musculoskeletal Research Department, Zeneca Pharmaceuticals, Mereside, Alderley \\ Park, Cheshire, SK10 4TG, United Kingdom
}

\begin{abstract}
Proteolytic degradation of aggrecan is a hallmark of the pathology of arthritis, yet the identity of the enzyme(s) in cartilage responsible for this degradation is unknown. Previous studies have suggested that the matrix metalloproteinases (MMPs) may be involved but there has been no definitive evidence for their direct action in the proteolysis of aggrecan in human arthritis.

We now show unequivocally that aggrecan fragments derived from the specific action of MMPs can be detected in synovial fluids from patients with both inflammatory and noninflammatory arthritis, with a neoepitope monoclonal antibody $\mathrm{AF}-28$ that detects the $\mathrm{NH}_{2}$-terminal sequence $F_{342}$ FGVG. . . . The synovial fluid MMP fragments were of low buoyant density and distributed exclusively at the top of cesium chloride density gradients, suggesting that these fragments lacked chondroitin sulfate chains. AF-28 immunoblotting of synovial fluid aggrecan fragments revealed a population of small AF-28 fragments of 30-50 kD. Based on their size relative to characterized products of an MMP-8 digest (Fosang, A.J., K. Last, P. Gardiner, D.C. Jackson, and L. Brown. 1995, Biochem. J. 310:337-343), these AF-28 fragments were derived from proteinase cleavage at, or near, the . . ITEGE IT3 $_{373} \downarrow$ ARGSV . . aggrecanase site. Immunodetection with polyclonal anti-ITEGE antiserum revealed that these fragments lacked the ... ITEGE ${ }_{374} \mathrm{COOH}$ terminus and were not therefore products of aggrecanase action. The same fluid samples contained a broad 6890-kD G1 fragment that contained the $\mathrm{COOH}-$ terminal . . . ITEGE IT4 $_{3}$ neoepitope. The results suggest that in some circumstances, despite extensive proteolysis of the core protein, aggrecan molecules may be cleaved by MMPs or aggrecanase in the interglobular domain, but not both. (J. Clin. Invest. 1996. 98:2292-2299.) Key words: proteinase - collagenase $\cdot$ proteoglycan $\bullet$ synovial fluid $\bullet$ arthritides
\end{abstract}

Address correspondence to Dr. Amanda Fosang, M.D., University of Melbourne, Department of Pediatrics, Orthopedic Molecular Biology Research Unit, Royal Children's Hospital, Parkville, 3052, Australia. Phone: 61-3-9345-6628; FAX: 61-3-9345-6668; E-mail: fosang@cryptic.rch.unimelb.edu.au

Received for publication 29 March 1996 and accepted in revised form 29 August 1996.

J. Clin. Invest.

(C) The American Society for Clinical Investigation, Inc. 0021-9738/96/11/2292/08 \$2.00

Volume 98, Number 10, November 1996, 2292-2299

\section{Introduction}

Joint damage and cartilage destruction are the common endpoint of human arthritis. The precise mechanisms involved are unknown but are likely to be complex and diverse. Aggrecan is the major proteoglycan in cartilage and the molecule that allows the tissue to bear weight by deforming elastically under compressive load. Proteinase degradation of aggrecan results in its release from the tissue which eventually leads to loss of joint function. The depletion of aggrecan from cartilage matrix in human and experimental arthritis is readily observed histologically by loss of metachromatic staining. The aggrecan fragments released from the tissue can be detected in synovial fluids of patients and experimental animals by immunoassay of specific protein (1-8) or carbohydrate $(7,9-13)$ epitopes on aggrecan molecules, by chemical assay of sulfated glycosaminoglycans $(8,13,14)$ or by isolation and sequencing of purified fragments $(15,16)$.

A large number of studies over more than a decade have shown that agents such as IL- $1 \alpha$, IL- $1 \beta$, TNF- $\alpha$, and retinoic acid promote cartilage resorption in vitro, and IL-1 has also been shown to promote aggrecan loss in vivo $(17,18)$. These agents are known to upregulate expression of some matrix metalloproteinase (MMP) ${ }^{1}$ genes in chondrocytes, suggesting a link between MMP expression and aggrecan degradation. Specific MMP inhibitors have also been shown to prevent loss of aggrecan from cartilage explant cultures in vitro (19-22). Together these studies have suggested that increased aggrecan degradation may be a consequence of upregulated MMP expression. However there has been no evidence for the direct degradative action of MMPs on aggrecan in human arthritis, largely because until recently efficient techniques for identifying proteolytic cleavage at the molecular level have not been available.

The development of neoepitope antibodies that detect newly created $\mathrm{NH}_{2}$ - or $\mathrm{COOH}$-terminal sequences resulting from specific proteolytic events has overcome this limitation. The first aggrecan neoepitope antibodies were described by Hughes et al. (23) and since then a number of neoepitope antibodies have been produced (24-26). One study has recently shown that an aggrecan G1 domain resulting from MMP action can be immunolocalized in cartilage sections from mice with experimentally induced arthritis using a neoepitope antibody (27).

The human MMPs cleave the aggrecan interglobular domain at . . DIPEN $341 \downarrow$ FFGVG. . . (28-31). Cleavage at this

1. Abbreviations used in this paper: $\mathrm{GuHCl}$, guanidinium hydrochloride; IB, incubation buffer; JCA, juvenile chronic arthritis; MMP, matrix metalloproteinase; OA, osteoarthritis; RA, rheumatoid arthritis. 
site in vivo would result in release into synovial fluid of fragments with $\mathrm{F}_{342} \mathrm{FGVG}$. . . $\mathrm{NH}_{2}$ termini. However, sequencing experiments have shown that the predominant high density aggrecan fragments found in human synovial fluids $(15,16)$ and in the medium of cartilage explant cultures (32-34) contain an $\mathrm{A}_{374}$ RGSVI. . . $\mathrm{NH}_{2}$-terminal sequence, demonstrating that cleavage at . . ITEGE $\downarrow \mathrm{A}_{374}$ RGSV . . is an important catabolic event. The enzyme responsible for cleavage at this site has been named aggrecanase but its identity remains unknown. At present there are no quantitative assays for measuring the ARGSV... neoepitope and its real abundance in tissue or fluids is not precisely known.

In this paper we provide unequivocal evidence that MMPs act directly in aggrecan degradation in arthritis. Fragments containing the $\mathrm{F}_{342} \mathrm{FGVG}$. . neoepitope have been detected in human synovial fluids by Western analysis and quantitated by competition elisa using the anti-FFGVG. . . neoepitope monoclonal, AF-28. The identification and partial characterization of AF-28 fragments sheds new light on possible mechanisms involved in aggrecan turnover and the relationship between MMP and aggrecanase activities in vivo.

\section{Methods}

Materials. Cysteine proteinase inhibitor E-64, pepstatin, and 2,2'Azino-di-[3 ethylbenzthiazoline sulfonate] (ABTS) were from Boehringer Mannheim (Mannheim, Germany). Keratanase (Pseudomonas sp.) (EC 3.2.1.103), and chondroitin ABC lyase (Proteus vulgaris) (EC 4.2.2.4) were from Seikagaku (Kogyo, Japan). Hyaluronidase (Streptomyces hyalurolyticus) (EC 4.2.2.1) and papain (EC 3.4.22.2) were from Sigma Chemical Co. (St. Louis, MO). [4-(2-aminoethyl) benzene sulfonylfluoride (AEBSF) was from CalbiochemNovabiochem Pty., (New South Wales, Australia). HiTrap desalting columns and Q-Sepharose were from Amrad Pharmacia Biotech, Melbourne, Australia. Rabbit anti-mouse horseradish peroxidase conjugated immunoglobulin and swine anti-rabbit horseradish peroxidase conjugated immunoglobulin were from DAKOPATTS (Copenhagen, Denmark). Immulon-4 $\mu$ l plates were from Dynatech Laboratories, Inc. (Chantily, VA). Monoclonal antibody 5-D-4 was a gift from Prof. Bruce Caterson (University of Cardiff, Wales, United Kingdom). Polyclonal anti-human G1 domain rabbit antiserum was a gift from Prof. Tim Hardingham (University of Manchester, Manchester, United Kingdom).

Patients. 68 synovial fluid samples were obtained from the knee joints of 47 patients attending Rheumatology Clinics who required either diagnostic or therapeutic aspiration. Diagnoses of rheumatoid arthritis (RA), osteoarthritis (OA), and Reiter's disease were based on the criteria recommended by the American Rheumatism Association (35-37). The diagnosis of other arthropathies was based on clinical presentation and serological and radiographic examination.

Collection, enzyme treatment, and analysis of synovial fluids. Complete aspirations of joint fluid were performed under aseptic conditions, without anesthetic, using a $1.2 \mathrm{~mm}$-bore needle. The fluids were collected into sterile tubes, centrifuged within $1 \mathrm{~h}$ to remove cells and stored frozen at $-20^{\circ} \mathrm{C}$. Some patients' joints were aspirated on more than one occasion during the sampling period (March 1994June 1995). For the detection of sulfated glycosaminoglycans in synovial fluids, $100-\mu \mathrm{l}$ aliquots were digested overnight at $60^{\circ} \mathrm{C}$ with 56 $\mu \mathrm{g}$ papain in buffer containing $10 \mathrm{mM}$ EDTA, $10 \mathrm{mM}$ cysteine, $0.2 \mathrm{M}$ sodium acetate, $\mathrm{pH} 5.8$, in a total volume of $120 \mu \mathrm{l}$. After digestion the samples were boiled for $30 \mathrm{~min}$ to inactivate the papain. For the detection of AF-28 epitope in synovial fluids, 500- $\mu$ l aliquots were digested overnight with shaking at $37^{\circ} \mathrm{C}$ with $5 \mathrm{U} / \mathrm{ml}$ hyaluronidase in the presence of the following proteinase inhibitors, specific for each class of proteinase: $20 \mu \mathrm{g} / \mathrm{ml} \mathrm{E} 64,1.25 \mathrm{mM}$ AEBSF, $5 \mathrm{mM}$ pepstatin, and $9.6 \mathrm{mM}$ EDTA. After digestion any precipitate was removed by centrifugation and the supernatants were dialyzed against distilled water. The strength of association between the concentration of AF-28 epitope and the concentration of sulfated glycosaminoglycan in the synovial fluids was measured using Spearman's rank correlation coefficient which is invariant to values assumed when data points are below detectable limits (38).

Competition assay for detecting the FFGVG. . neoepitope with monoclonal AF-28. The standard and coating antigen used in the assay was a 32-amino acid synthetic peptide with the sequence FFGVGGEEDITVQTVTWPDMELPLPRNITEGE. This is part of the amino acid sequence present in the human aggrecan interglobular domain (39), where FFGVG. . represents the $\mathrm{NH}_{2}$ terminal sequence generated by the action of matrix metalloproteinases, and . . . ITEGE represents the $\mathrm{COOH}$-terminal sequence generated by the action of the putative aggrecanase enzyme. The peptide synthesis and assay procedure have been described previously (26). Briefly, microtitre plates were coated overnight at $4^{\circ} \mathrm{C}$ with $25 \mathrm{pmol} / \mathrm{ml} 32$-mer peptide. The coating solution was removed and the plates were washed six times in a Tris incubation buffer (Tris IB) containing $0.1 \%$ (wt/vol) BSA, $0.1 \%$ (wt/vol) Nonidet NP-40, $0.15 \mathrm{M} \mathrm{NaCl}$, and $10 \mathrm{mM}$ Tris$\mathrm{HCl}, \mathrm{pH} 7.4$, and then blocked for $1 \mathrm{~h}$ at $37^{\circ} \mathrm{C}$ with $1 \%$ BSA in Tris IB. After washing, samples or standard $(100 \mu \mathrm{l})$ were added to the wells, followed by $100 \mu \mathrm{l}$ of AF-28 antibody $(1: 1,000)$ and incubated for $1 \mathrm{~h}$ at $37^{\circ} \mathrm{C}$. The plates were washed and incubated with an antimouse antibody conjugated with horseradish peroxidase $(1: 1,000)$ for $1 \mathrm{~h}$ at $37^{\circ} \mathrm{C}$. After washing, the peroxidase substrate ABTS was added and the plates were incubated at room temperature during color development. Progress of the color development was monitored at 405 $\mathrm{nm}$ till the optical density in the wells containing antibody with no competitor (maximum binding) reached $>1.2$. The limit of detection for the assay was $7 \mathrm{pmol} / \mathrm{ml}$.

Dye binding assay for detecting sulfated glycosaminoglycans. An aliquot of each synovial fluid sample was digested with papain prior to assay for sulfated glycosaminoglycan by the 1,9-dimethylmethylene blue assay (40) modified to suit a microplate assay (13).

Fractionation of synovial fluids by cesium chloride density gradient centrifugation. Nine synovial fluid samples were diluted 1:2 with $8 \mathrm{M}$ guanidine hydrochloride $(\mathrm{GuHCl})$ and adjusted to a density of $1.5 \mathrm{~g} / \mathrm{ml}$ by the addition of solid cesium chloride. The samples were centrifuged at $34,000 \mathrm{rpm}$ for $20 \mathrm{~h}$ at $10^{\circ} \mathrm{C}$ in a Beckman Vti 50 vertical rotor. The resulting gradients were divided into five equal fractions designated D1 at the bottom $(\delta>1.5)$ and D5 at the top $(\delta<$ 1.4). The density of each fraction was measured prior to extensive dialysis against distilled water and freezedrying.

Fractionation of synovial fluids by $Q$-Sepharose chromatography. Aggrecan fragments present in synovial fluids were partially purified on Q-Sepharose as described by Ilic et al. (33). Fluids from two patients, one with $\mathrm{OA}(7.5 \mathrm{ml})$ and one with seronegative inflammatory arthritis $(15 \mathrm{ml})$, were digested overnight with hyaluronidase. The samples were diluted to $100 \mathrm{ml}$ in buffer containing $6 \mathrm{M}$ urea, $0.15 \mathrm{M} \mathrm{NaCl}, 50 \mathrm{mM}$ sodium acetate, $\mathrm{pH} 6.0$, and applied to $2 \mathrm{ml}$ Q-Sepharose columns in which the outflow tubing was looped back into the column sample reservoir. The columns were pumped at 0.3 $\mathrm{ml} / \mathrm{min}$ overnight allowing the fluid samples to cycle through the column three times. Unbound material was not analyzed further because AF-28 fragments were assumed to contain keratan sulfate chains at $\mathrm{Thr}_{352}$ and $\mathrm{Thr}_{357}$ in the IGD (41) and would therefore bind to the column. Material that bound to the column was eluted firstly with buffer containing $6 \mathrm{M}$ urea, $0.5 \mathrm{M} \mathrm{NaCl}, 50 \mathrm{mM}$ sodium acetate, $\mathrm{pH} 6.0$, and subsequently with buffer containing $4 \mathrm{M} \mathrm{GuHCl}, 0.5 \mathrm{M}$ $\mathrm{NaCl}, 50 \mathrm{mM}$ sodium acetate, $\mathrm{pH} 6.0$, with monitoring at $278 \mathrm{~nm}$. Fractions containing proteins that eluted with $6 \mathrm{M}$ urea/ $/ .5 \mathrm{M} \mathrm{NaCl}$ or $4 \mathrm{M} \mathrm{GuHCl} / 0.5 \mathrm{M} \mathrm{NaCl}$ were pooled and applied to HiTrap desalting columns equilibrated with $50 \mathrm{mM}$ ammonium hydrogen carbonate. The excluded volume fractions were pooled, freeze-dried and reconstituted in $300 \mu \mathrm{l}$ water, and digested with $0.01 \mathrm{U} / \mathrm{ml}$ chondroitinase $\mathrm{ABC}$ and $0.01 \mathrm{U} / \mathrm{ml}$ keratanase overnight at $37^{\circ} \mathrm{C}$ before analysis by SDS-PAGE. 
Table I. Details of Number and Type of Arthritis Patients

\begin{tabular}{|c|c|c|c|}
\hline Arthropathy & $\begin{array}{l}\text { Number } \\
\text { of patients }\end{array}$ & $\begin{array}{l}\text { Total number } \\
\text { of samples } \\
\text { taken }\end{array}$ & Female/Male \\
\hline Osteoarthritis & 4 & 8 & $4 / 0$ \\
\hline Rheumatoid arthritis & 14 & 22 & $11 / 3$ \\
\hline Juvenile chronic arthritis & 17 & 17 & $11 / 6$ \\
\hline Psoriatic juvenile chronic arthritis & 2 & 4 & $2 / 0$ \\
\hline Psoriatic arthritis & 2 & 2 & $1 / 1$ \\
\hline Chron's arthropathy & 1 & 2 & $1 / 0$ \\
\hline Crystal-type arthritis & 1 & 1 & $0 / 1$ \\
\hline Systemic lupus erythromatosis & 1 & 1 & $1 / 0$ \\
\hline Reiter's disease & 1 & 1 & $1 / 0$ \\
\hline Seronegative inflammatory arthritis & 3 & 9 & $3 / 0$ \\
\hline Mixed connective tissue disease & 1 & 1 & $1 / 0$ \\
\hline Total & 47 & 68 & $36 / 11$ \\
\hline
\end{tabular}

Analysis of partially purified aggrecan fragments by SDS-PAGE and immunoblotting. Deglycosylated aggrecan fragments partially purified by Q-Sepharose chromatography were electrophoresed on $5 \%$ SDS-polyacrylamide gels (42) and transferred to immobilon for Western blot analysis. Samples for immunodetection with AF-28, the antikeratan sulfate antibody 5-D-4 $(43,44)$ and anti-G1 domain $(45)$ were electophoresed unreduced. Samples for immunodetection with the neoepitope anti-ITEGE antiserum RAM 3-2 (46) and the preimmune serum RAM 3-0 were reduced by heating with 5\% mercaptoethanol for $30 \mathrm{~min}$ at $60^{\circ} \mathrm{C}$.

\section{Results}

Synovial fluids from patients with a range of different arthritides (Table I) were assayed for the presence of the AF-28 epitope (Fig. $1 A$ ) which reveals MMP-released aggrecan fragments, and sulfated glycosaminoglycan (Fig. $1 \mathrm{~B}$ ) which detects total released aggrecan fragments. 68 individually dated samples were assayed and 37 of these contained detectable levels of AF-28 epitope (Fig. $1 A$ ). Fluids taken from patients with OA, RA, juvenile psoriatic arthritis (JCA), crystal-type arthritis, Reiter's disease, and seronegative inflammatory arthritis contained detectable levels of AF-28 epitope, indicating that MMP cleavage at ...DIPEN $N_{341} \downarrow$ FFGVG. . . occurs in all the major arthritides. The concentrations of sulfated glycosaminoglycan detected in the fluids (Fig. $1 B$ ) were in a similar range to those reported previously $(8,13)$.

The relationship between the concentration of AF-28 epitope and sulfated glycosaminoglycan in synovial fluid of patients with OA, RA, JCA, and inflammatory arthritis was analyzed using the Spearman's rank correlation coefficient (Fig. 2). A statistically significant correlation between AF-28 epitope and total glycosamioglycan was found in OA patients $\left(R_{\mathrm{S}}=0.7425 ; P=0.0349\right)$. There was no correlation in patients with RA, JCA, and inflammatory arthritis suggesting that the ratio of AF-28 epitope/glycosaminoglycan is not constant or similar in the inflammatory diseases and that treatment regimes or stage of disease may influence the concentration of aggrecan components in synovial fluids. In the future it will be useful to correlate the changing patterns in AF-28 and glycosaminoglycan levels with other parameters of the inflammatory process.

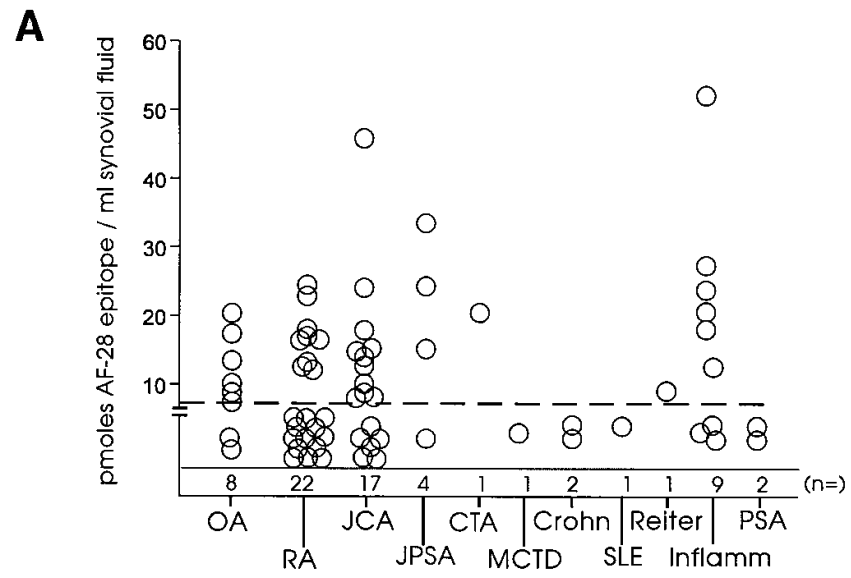

B

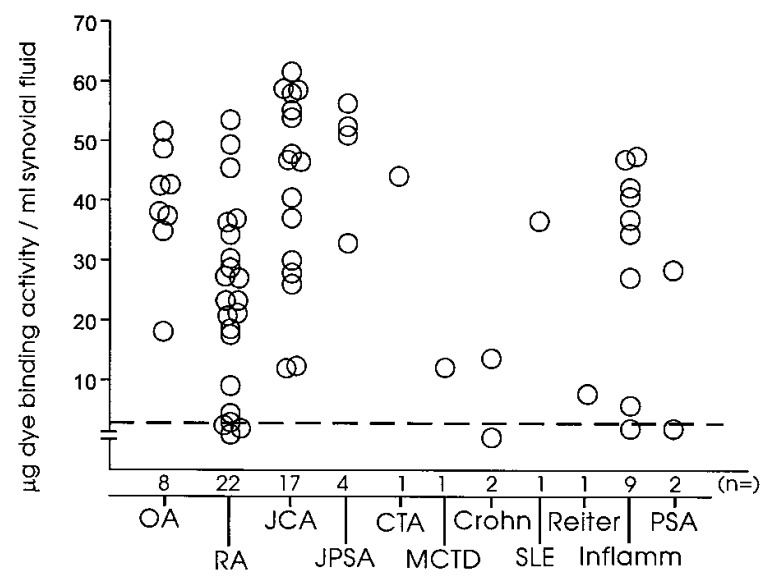

Figure 1. Concentration of AF-28 epitope and sulfated glycosaminoglycan in human synovial fluid. $(A)$ Aliquots of human synovial fluid were digested with hyaluronidase prior to assay for AF-28 epitope by competition ELISA or $(B)$ digested with papain prior to assay for sulfated glycosaminoglycan by the 1,9-dimethylmethylene blue assay. $O A$, osteoarthritis; $R A$, rheumatoid arthritis; $J C A$, juvenile chronic arthritis; $J P S A$, juvenile psoriatic arthritis; $C T A$, crystaltype arthritis; $M C T D$, mixed connective tissue disease; Crohn, Crohn's arthropathy; SLE, systemic lupus erythromatosis; Reiter, Reiter's disease; Inflamm, seronegative inflammatory arthritis; and $P S A$, psoriatic arthritis.

Presumably, many of the aggrecan fragments in synovial fluids that do not contain AF-28 epitope will contain the ARGSV. . . $\mathrm{NH}_{2}$-terminal sequence generated by aggrecanase cleavage. These fragments are relatively large and composed of a segment of the IGD attached to the G2 domain, the KS domain, and variable lengths of the chondroitin sulfate domain. Because of their large size and high buoyant density, these aggrecanase fragments have been recovered at the bottom of cesium chloride density gradients $(15,16)$. It was of interest to analyse the distribution of AF-28 fragments in cesium chloride density gradients because we have shown previously (26) that some AF-28 epitope in synovial fluids is present on fragments that are of a comparatively smaller size.

Synovial fluids from patients with seronegative inflammatory arthritis (three samples), Crohn's disease, OA, RA, psoriatic arthritis, and JCA (two samples) were fractionated into five equal fractions on cesium chloride density gradients and analyzed for AF-28 epitope (data not shown). For each patient 
A

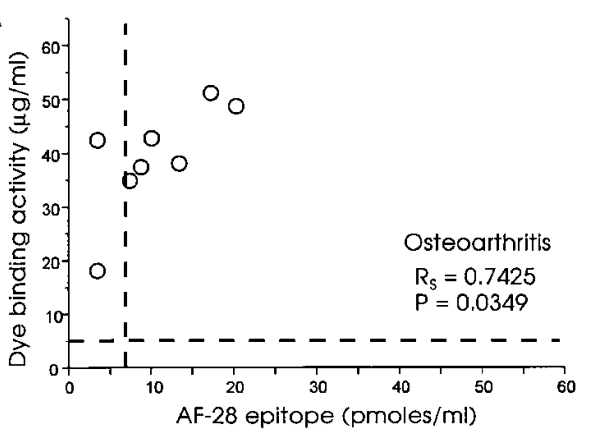

C

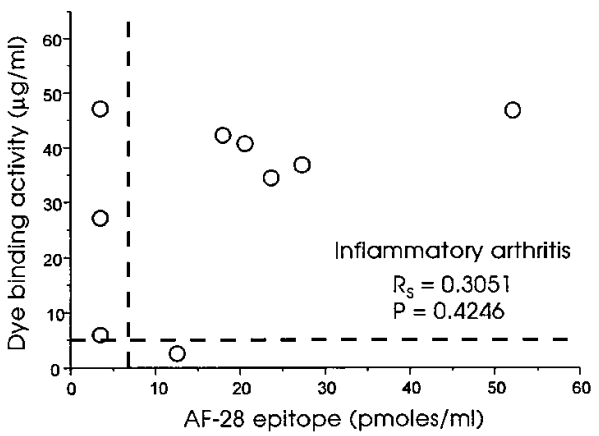

B

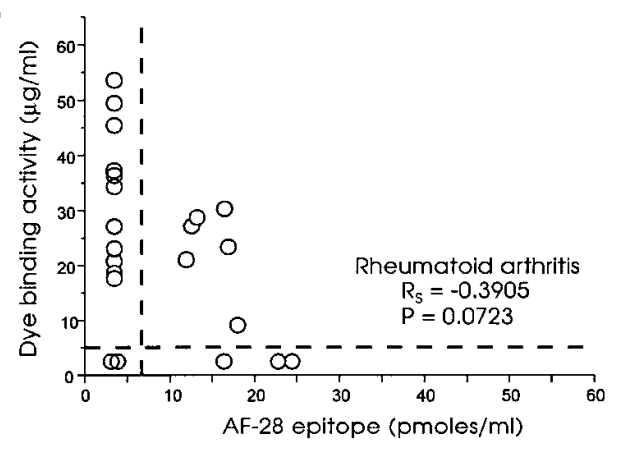

D

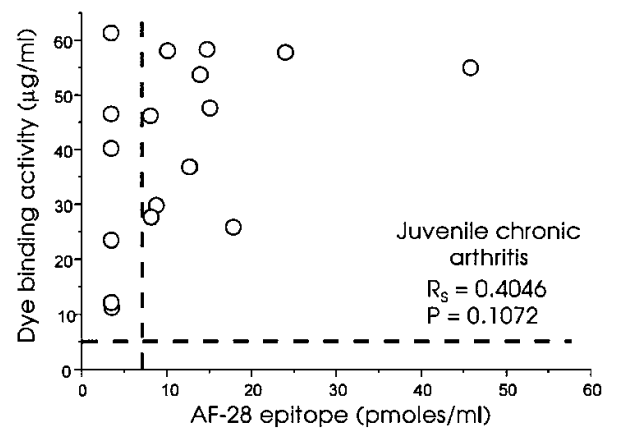

Figure 2. Relationship between the concentration of AF-28 epitope and sulfated glycosaminoglycans in human synovial fluids. The concentration of AF-28 epitope (pmoles/ml synovial fluid) is plotted against the concentration of sulfated glycosaminoglycan, measured as dye binding activity ( $\mu \mathrm{g} / \mathrm{ml}$ synovial fluid) for patient groups with either OA $(A)$, RA $(B)$, inflammatory arthritis $(C)$, or JCA $(D)$. The correlation coefficients $\left(R_{\mathrm{S}}\right)$ and $P$ values are given. fluid, AF-28 epitope was recovered at the top of the density gradient in the D5 fractions. No AF-28 epitope was measured in the D1, D2, D3, or D4 fractions demonstrating that fragments with FFGVG. . . $\mathrm{NH}_{2}$ termini were of low buoyant density, and further demonstrating that these fragments lacked chondroitin sulfate chains and contained only a small portion, or none, of the keratan sulfate-rich region (47). The results therefore suggest that $\mathrm{COOH}$-terminal processing of all

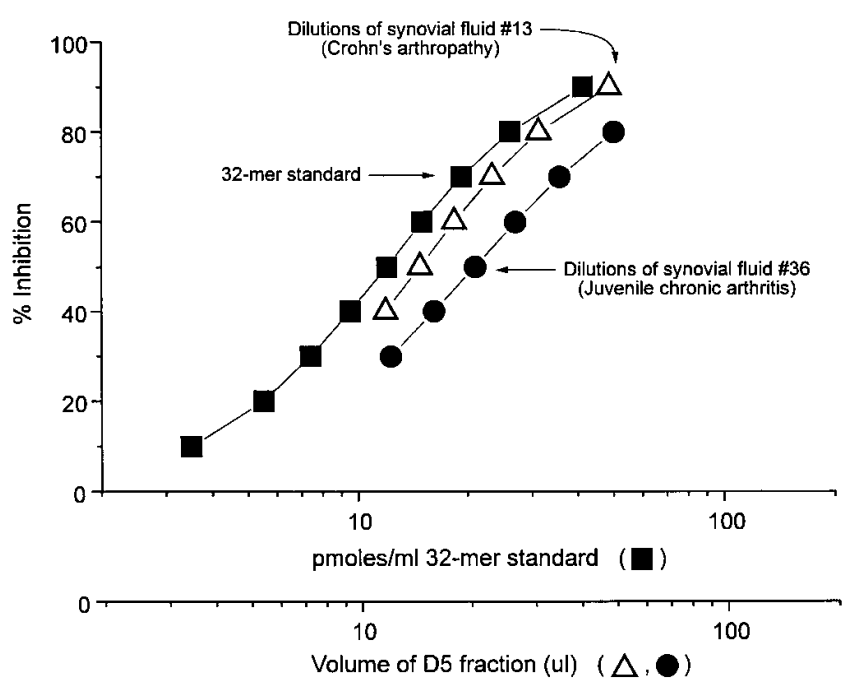

Figure 3. Specific binding of AF-28 antibody to low buoyant density aggrecan fragments. Doubling dilutions of the protein-rich D5 fractions obtained by cesium chloride density gradient centrifugation of synovial fluids from a patient with juvenile chronic arthritis $(\bullet)$ or Crohn's arthropathy $(\triangle)$ were analyzed in the AF-28 competition ELISA. The competition curves generated by the synovial fluids are parallel with the standard curve (ם).
MMP-derived fragments occurs in vivo and few, if any, chondroitin sulfate-containing MMP fragments remain in the synovial fluid. Alternatively, there may be a population of truncated aggrecan molecules accumulated in the tissue which have already lost their chondroitin sulfate chains due to proteolytic processing of the core protein, and these truncated molecules may be preferred substrates for the MMPs. Given that none of the nine fluids contained epitope in the D1-D4 fractions, a $95 \%$ confidence interval for the proportion in the population that might contain AF-28 epitope in the D1-D4 fractions does not rule out values between $0-28 \%$, while a $75 \%$ confidence interval would allow for $0-14 \%$ (48).

To eliminate the possibility that monoclonal AF-28 was binding nonspecifically to another component in the proteinrich D5 fraction of the gradients, dilutions of D5 fractions were assayed by competition ELISA (Fig. 3). AF-28 antibody binding specifically to dilutions of FFGVG. . . neoepitope would be expected to generate a competition curve with a slope parallel with the standard antigen. In contrast, nonspecific binding due to a weak and variable affinity of the antibody for other molecules would be expected to generate a nonparallel competition curve, or no curve at all. The inhibition curves generated from dilutions of synovial fluids were parallel with the standard antigen (Fig. 3). Thus, AF-28 epitope detected in the D5 fractions was specifically detecting aggrecan fragments with $\mathrm{NH}_{2}$-terminal FFGVG. . . sequences.

We have shown previously that a small 30-50-kD AF-28positive band can be found in unfractionated synovial fluids from some patients with arthritis, and based on its molecular size relative to characterized products of an MMP-8 digest (26) we postulated that this fragment was the product of cleavage at both the MMP site and the aggrecanase site. To test this hypothesis we have now used immunodetection with an antiITEGE antiserum, RAM 3-2 (46) to identify the COOH terminus of the $30-50 \mathrm{kD}$ fragment. Aggrecan fragments in sy- 

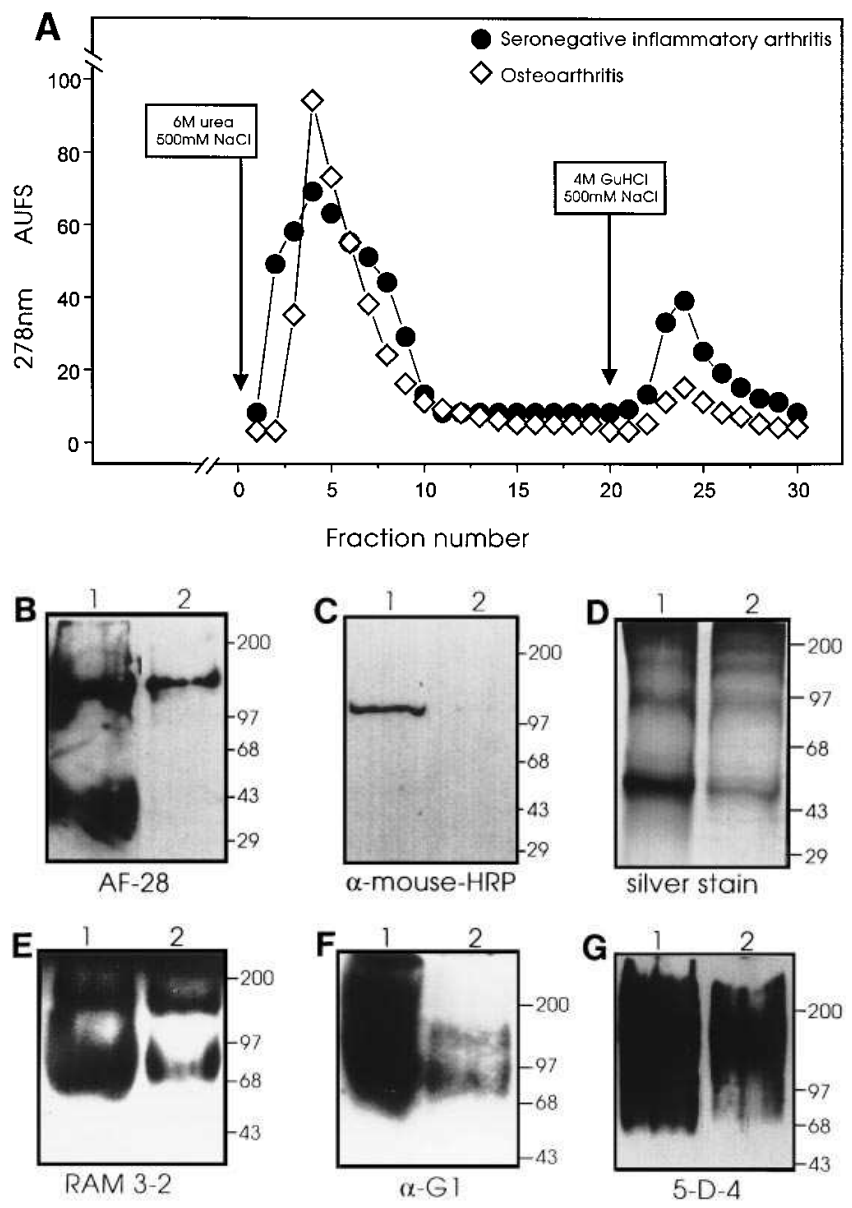

Figure 4. Partial characterization of a glycosylated IGD fragment with FFGVG. .. $\mathrm{NH}_{2}$ terminus. Hyaluronidase digested synovial fluid from patients with seronegative inflammatory arthritis $(\mathbf{0}$; lane 1$)$ and osteoarthritis $(\diamond$; lane 2$)$ were applied to Q-Sepharose and bound proteoglycans were eluted with $6 \mathrm{M}$ urea, $0.5 \mathrm{M} \mathrm{NaCl}$ followed by $4 \mathrm{M} \mathrm{GuHCl}, 0.5 \mathrm{M} \mathrm{NaCl}(A)$. Aliquots of aggrecan fragments present in the $4 \mathrm{M} \mathrm{GuHCl}$ eluted fractions were electrophoresed on $5 \%$ SDS gels and analyzed by silver stain $(D)$ or immunodetection with monoclonal AF-28 (B), anti-mouse-HRP $(C)$, RAM 3-2 $(E)$, anti-G1 $(F)$, and 5-D-4 $(G)$.

novial fluids from two arthritis patients were partially purified by Q-Sepharose chromatography (Fig. $4 A$ ), deglycosylated and analyzed by immunoblotting with AF-28 (Fig. $4 B$ ), RAM 3-2 antiserum specific for the $\mathrm{COOH}$ terminal . . ITEGE 374 neoepitope resulting from aggrecanase action (Fig. $4 E$ ), 5-D-4 (Fig. $4 G$ ) and anti-G1 domain antiserum (Fig. $4 F$ ). Material which was bound to the column and eluted with $6 \mathrm{M}$ urea/ $/ 0.5 \mathrm{M}$ $\mathrm{NaCl}$ did not contain any aggrecan epitopes (results not shown). The material which was bound to the column and eluted with $4 \mathrm{M} \mathrm{GuHCl}$ included two AF-28 positive bands of 30-50- and 120-150-kD and two RAM 3-2 bands of 68-80-kD and $\sim 150 \mathrm{kD}$. There was no RAM 3-2 band with 30-50 kD. Because the 30-50-kD AF-28 fragment did not appear to contain an . . ITEGE $373 \mathrm{COOH}$ terminus, this is a strong indication that it was not the product of aggrecanase action. The 120-150-kD band detected with AF-28 may represent an MMP fragment with an intact G2 domain (Fig. 5). The data suggest that in these patients the processing that occurred
COOH-terminal to the MMP site was the result of proteolysis by an enzyme(s) other than aggrecanase (Fig. 5). Some weak nonspecific staining at about $120 \mathrm{kD}$ was detected with an anti-mouse horseradish peroxidase conjugated immunoglobulin in one of the patient samples (Fig. $4 C$ ).

The RAM 3-2 immunoblots revealed two major bands with 68-90-kD and $\sim 150 \mathrm{kD}$. The $68-90-\mathrm{kD}$ aggrecanase band was identified as G1 domain by immunodetection with anti-human G1 antiserum (Fig. $4 F$ ). The size and COOH-terminal sequence of this G1 fragment indicated that it had not been cleaved at the MMP site and therefore retained an intact ...DIPENFFGVG. . . sequence (Fig. 5). The detection of a $150-\mathrm{kD}$ RAM 3-2 band was unexpected. Immunodetection of both the 68-90- and 150-kD RAM 3-2 bands was blocked by pre-incubation of the antibody with a 32-mer peptide with . . .ITEGE $\mathrm{COOH}$ terminus, confirming the specificity of the antibody (results not shown). It is possible that RAM 3-2 may also detect ...ELE $E_{1545}$ and/or $\mathrm{EEE}_{1714}$ neoepitopes in the chondroitin sulfate domain (49), as these fragments would be approximately $150 \mathrm{kD}$ after deglycosylation. Immunodetection with the preimmune serum RAM 3-0 did not show any nonspecific staining (results not shown). A spectrum of fragments containing G1 epitopes (Fig. $4 F$ ) and 5-D-4 epitope on keratan sulfate chains (Fig. $4 G$ ) were also detected.

\section{Discussion}

We recently showed by Western analysis that synovial fluids from four patients with arthritis contained material that was immunoreactive with a monoclonal antibody that detects MMP-derived aggrecan fragments. This finding encouraged us to further investigate the direct involvement of MMPs in aggrecan proteolysis since this had been postulated for human joint pathology, but not confirmed. The present paper shows that in about $50 \%$ (37 of 68) of arthritis patients, aggrecan is cleaved by MMPs and the MMP degradation products are released from the articular cartilage into the synovial space. The size (30-50 kD; 120-150 kD) and low buoyant density of the AF-28 fragments suggest that they are relatively short fragments that terminate within the IGD near the aggrecanase site, or within the keratan sulfate-rich region, as no high density fragments bearing chondroitin sulfate chains were detected in the gradient D1 fractions.

Several studies have demonstrated release of aggrecanase fragments in vivo $(15,16)$ and in vitro $(32-34,50)$ but there have been no reports of MMP-released fragments in human disease or animal models. The . . VDIPEN neoepitope on G1 fragments retained in the tissue following MMP cleavage has been immunolocalized in sections of human joint tissue (51) and in mice with collagen II-induced arthritis (27), however the corresponding FFGVG. . . neoepitope that is released into the joint space has not been reported. One reason why these MMP-released fragments have not been found in synovial fluids previously is that earlier studies isolated high-buoyant density aggrecan fragments from the bottom of cesium chloride density gradients. The results of the present study however show that all MMP-derived products with FFGVG. . . $\mathrm{NH}_{2}$ termini are recovered at the top of density gradients.

An estimate of the proportion of aggrecan molecules containing an FFGVG. . . $\mathrm{NH}_{2}$ terminus can be made be calculating picomoles of aggrecan present in each synovial fluid from the concentration of sulfated glycosaminoglycan, assuming for 
the purposes of the calculation that (a) sulfated glycosaminoglycan represents $90 \%$ of the mass of human aggrecan, and (b) that the relative molecular mass of human aggrecan is $1,000,000 \mathrm{D}$. The percentage of aggrecan molecules containing AF-28 epitope can then be estimated from pmol AF-28 epitope/pmol aggrecan. More than half of the patient fluids assayed contained detectable levels of epitope, and of these the proportion of aggrecan molecules that contained AF-28 epitope ranged from 20 to $100 \%$. In general AF-28 epitope was present on less than half the aggrecan population present in the fluids, but a small proportion (32\%) had more than $50 \%$ aggrecan with AF-28 epitope, and a minority (12\%) had equimolar amounts of aggrecan fragments and AF-28 epitope. These extremes highlight the diversity and complexity of aggrecan catabolism and suggest that in some cases MMPs may be wholly responsible for aggrecan cleavage in the IGD, while under other conditions MMPs may not be involved at all. The limitation of this calculation is the assumption of molecular size. Most aggrecan molecules are truncated at their $\mathrm{COOH}$ terminal end and the extent of truncation may be quite significant. Although these percentages represent estimated values on a small number of samples, they emphasize the point that MMP cleavage of aggrecan is not trivial.

In the absence of quantitative assays for measuring the concentration of all the relevant neoepitopes present in synovial fluids it is impossible to reconcile the relative extent of cleavage occurring at each of the important catabolic sites. It is clear however that the involvement of MMPs in aggrecan catabolism is significant, and may even be greater than suggested by the present results. One important piece of information that is lacking for both FFGVG. . . fragments and ARGSV. . . fragments is their rate of clearance from synovial joints. If FFGVG. . . fragments are cleared faster than ARGSV. . fragments, perhaps because they are smaller or do not carry chondroitin sulfate chains, then the calculation of AF-28 epitope per total chondroitin sulfate may give a minimum incidence of MMP activity. In addition the measurement of AF-28 epitope is limited by the sensitivity of the competition assay, which is

(a)

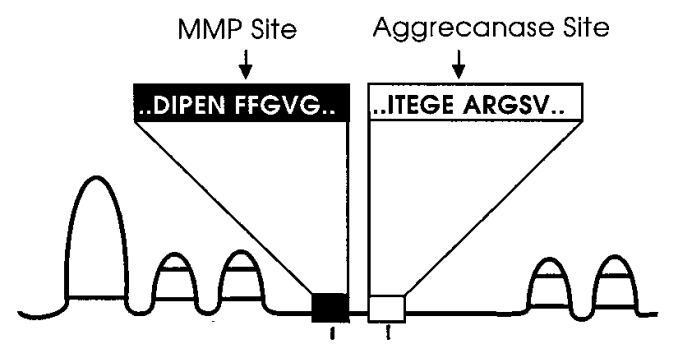

(b)

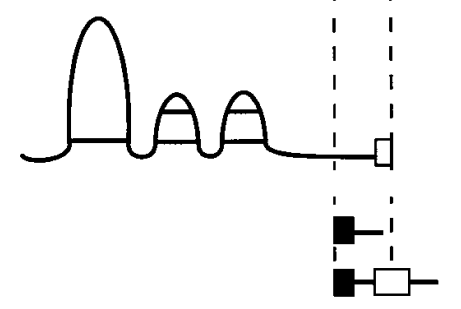

68-90 kD Gl fragment with ITEGE... COOH-terminus

(d) $120-150 \mathrm{kD} \quad \mathrm{G} 2$ fragment with FFGVG... $\mathrm{NH}_{2}$-terminus

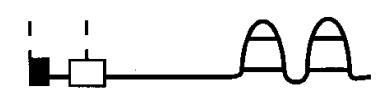

Figure 5. Schematic representation of G1, G2, and IGD fragments detected in human synovial fluids. comparatively less sensitive than a sandwich ELISA. One third of patient fluids assayed did not contain detectable levels of AF-28 epitope but it is entirely possible that epitope could be detected in these patients with a more sensitive sandwich assay.

The immunodetection studies (Fig. 4) refute an earlier hypothesis that a $30-50-\mathrm{kD}$ fragment with an FFGVG. . . $\mathrm{NH}_{2}$ terminus was the product of MMP and aggrecanase cleavage in the IGD and contained a COOH-terminal . . .ITEGE sequence. The small size of this fragment however indicates that the $\mathrm{COOH}$-terminal cleavage site is in close proximity to the aggrecanase site. We have shown that MMP-3 (52) and MMP-13 (31) cleave within 4 and 11 residues of the aggrecanase site, respectively. Plasmin, urokinase (53), and elastase (54) are also known to cleave the IGD in this proteinase-sensitive region. Given that at least seven different G1 fragments resulting from multiple cleavage points and/or multiple proteinases, can be detected in extracts of human articular cartilage (55), it would not be surprising to find that multiple cleavage sites in the IGD are also utilized during aggrecan turnover.

The immunodetection studies question the supposition that aggrecanase activity is always present. Rather, the results suggest that in some cases, aggrecan molecules cleaved by MMPs at . . .DIPEN $\downarrow$ FFGVG. . . are not cleaved further by aggrecanase, but are subject to proteolysis by other proteinases or MMPs. This is an interesting and important observation that has not been made before. Relative to the MMPs, aggrecanase was active on a different population of aggrecan molecules since G1 fragments with . . .ITEGE COOH termini were detected in the same fluid sample. Thus aggrecanase and MMPs could be present at different microanatomical sites within cartilage. This is consistent with immunolocalization studies showing that ... .DIPEN and . . .ITEGE neoepitopes were often noncoincident within a single metatarsal joint of CIA mice (56). In contrast the codistribution of . . . DIPEN and . . .ITEGE epitope in other situations (57) argues that MMPs and aggrecanase are colocalized but that aggrecanase fragments resist MMP cleavage. Our results now extend these observations and suggest that MMP fragments may also resist aggrecanase cleavage. It is possible that truncated aggrecan molecules that have been extensively processed from their $\mathrm{COOH}$-terminal ends and which have accumulated in the tissue over time may represent preferred substrates for MMPs, while longer aggrecan molecules are preferentially cleaved by aggrecanase. This model would explain the small size of the AF-28 fragments found in human synovial fluid.

There is another possible scenario that may account for the small size of the FFGVG. . . fragments: following MMP cleavage and release of FFGVG. . .-containing fragments from the tissue, MMPs or other proteinases secreted by cells in the synovium degrade the fragments further. However, in the synovial fluid from patients with all arthritides except septic arthritis $(58,59)$ there is an excess of TIMP and $\alpha_{2}$-macroglobulin, and all endogenous active MMPs would be expected to be totally inactivated. At present we cannot distinguish between proteolysis in cartilage and proteolysis in synovial fluid but we do not believe MMP action is significant in the synovial fluid. In addition if there was extensive proteolysis occurring in synovial fluid, it is difficult to imagine why MMP-released fragments would be cleaved into smaller products of low buoyant density, while aggrecanase-released fragments resist cleavage and remain large and of high buoyant density. We also cannot 
exclude two possible reasons why RAM 3-2 did not detect the $30-50-\mathrm{kD}$ AF-28 fragment. Firstly that the 30-50-kD fragment is cleaved by aggrecanase but that a carboxy peptidase in synovial fluid removes the $\mathrm{COOH}$-terminal neoepitope, or secondly that the ...ITEGE COOH terminus of the polypeptide is bound to the membrane and not available for antibody binding.

The RAM 3-2 antibody used in this study has been characterized and shown to specifically detect the . . ITEGE neoepitope (46). In the present study the Western blot results suggest that RAM 3-2 also detects an epitope present on fragments larger than the G1 domain, with a molecular weight of $\sim 150,000$. The most likely interpretation of this finding is that the ...ELE $E_{1545}$ and/or EEE $E_{1714}$ neoepitopes in the chondroitin sulfate domain (49) are also detected by the antiserum, because immunoreactivity was blocked by preincubation of RAM 3-2 with an . . ITEGE 32-mer synthetic peptide. The high charge and spatial arrangement of the glutamate residues (...ExE) are likely to be major antigenic determinants for the epitope. It is important therefore to be aware of the potential cross-reactivity of Ram 3-2 and other polyclonal anti-ITEGE antisera when observing immunostaining for aggrecanase neoepitopes.

The biochemical relationship between MMP and aggrecanase activities remains obscure, as does the biological advantage that might be achieved by locating two catalytic sites in such close proximity. There are no clues as to why this should be so, and no clues as to the identity of the putative aggrecanase. One member of the MMP family, MMP-8, has been shown to have aggrecanase activity in vitro (52). MMP- 8 mRNA is expressed in human fetal chondrocytes (Fosang, A.J., J. Dudhia, and $\mathrm{M}$. Vitellone, unpublished results), normal, and OA cartilage (60) and is upregulated by IL-1 $(61,62)$. However because its mode of action in vitro is strikingly different to aggrecanase action in cell and explant culture it is unclear whether this MMP could be responsible for aggrecanase activity in vivo. The action of MMP-8 on an aggrecan G1-G2 substrate in vitro is to cleave primarily at the MMP site, and only subsequently at the aggrecanase site (52). In contrast, turnover of aggrecan by cultured rat chondrosarcoma cells and primary bovine chondrocytes is mediated almost exclusively by aggrecanase (63). A model which proposes that in vivo MMP-8 or an MMP-8-like enzyme is responsible for aggrecanase action, must also explain how the specificity of the enzyme for sites on the substrate can switch, and under what conditions. The challenge for the future is to ascertain the relative abundance of the different catabolic neoepitopes of aggrecan and to determine the relative importance of MMP cleavage and aggrecanase cleavage in normal cartilage and in arthritis.

\section{Acknowledgments}

We are indebted to Dr. Roger Allen (Royal Children's Hospital, Melbourne, Australia) and Drs. David Barraclough, Andrea Bendrups, Geoff McColl, and Ian Wicks (Royal Melbourne Hospital, Melbourne, Australia) for collecting synovial fluids from their patients. We thank Assoc. Prof. Terry Nolan and Dr. John Carlin (Department of Clinical Epidemiology and Biostatistics, Royal Children's Hospital) for advice on the statistical analyses. We thank Prof. Tim Hardingham (University of Manchester, Manchester, UK) and Prof. Bruce Caterson (University of Cardiff, Cardiff, United Kingdom) for generously providing antibodies for this study.
We gratefully acknowledge financial support from the National Health and Medical Research Council of Australia and the Arthritis Foundation of Australia.

\section{References}

1. Heinegård, D., S. Inerot, J. Wieslander, and G. Lindblad. 1985. A method for the quantification of cartilage proteoglycan structures liberated to the synovial fluid during developing degenerative joint disease. Scand. J. Clin. Lab. Invest. 45:421-427.

2. Saxne, T., D. Heinegård, F.A. Wollheim, and H. Pettersson. 1985. Differences in cartilage proteoglycan level in synovial fluid in early rheumatoid arthritis and reactive arthritis. Lancet. 20:127-128.

3. Witter, J., P.J. Roughley, C. Webber, N. Roberts, E. Keystone, and A.R. Poole. 1987. The immunologic detection and characterization of cartilage proteoglycan degradation products in synovial fluids of patients with arthritis. Arthritis Rheum. 30:519-529.

4. Saxne, T., D. Heinegård, and F.A. Wollheim. 1986. Therapeutic effects on cartilage metabolism in arthritis as measured by release of proteoglycan structures into the synovial fluid. Ann. Rheum. Dis. 45:491-497.

5. Saxne, T., and D. Heinegård. 1992. Synovial fluid analysis of two groups of proteoglycan epitopes distinguishes early and late cartilage lesions. Arthritis Rheum. 35:385-390.

6. Saxne, T., F. Castro, U. Rydholm, and H. Svantesson. 1989. Cartilage derived proteoglycans in body fluids of children. Inverse correlation with age. $J$. Rheumatol. 16:1341-1344.

7. Poole, A.R., M. Ionescu, A. Swan, and P.A. Dieppe. 1994. Changes in cartilage metabolism in arthritis are reflected by altered serum and synovial fluid levels of the cartilage proteoglycan aggrecan. J. Clin. Invest. 94:25-33.

8. Carroll, G. 1989. Measurement of sulfated glycosaminoglycans and proteoglycan fragments in arthritic synovial fluid. Ann. Rheum. Dis. 48:17-24.

9. Shimozuru, Y., M. Shinmei, T. Naraki, Y. Iwasaki, M.E. Lenz, and E.J.M.A. Thonar. 1995. Rapid and sensitive sandwich enzyme immunoassay for the quantification of antigenic keratan sulfate in body fluids. Trans. Orthop. Res. Soc. 20:419.

10. Thonar, E.J.-M.A., M.E. Lenz, G.K. Klintworth, B. Caterson, L.M. Pachman, P. Glickman, R. Katz, J. Huff, and K.E. Kuettner. 1985. Quantification of keratan sulfate in blood as a marker of cartilage catabolism. Arthritis Rheum. 28:1367-1376.

11. Ratcliffe, A., W. Shurety, and B. Caterson. 1993. The quantitation of a native chondroitin sulfate epitope in synovial fluid lavages and articular cartilage from canine experimental osteoarthritis and disuse atrophy. Arthritis Rheum. 36:543-551.

12. Carlson, C.S., R.F. Loeser, B. Johnstone, H.M. Tulli, D.B. Dobson, and B. Caterson. 1995. Osteoarthritis in cynomolgus macaques. II Detection of modulated proteoglycan epitopes in cartilage and synovial fluid. J. Orthop. Res. 13:399-409.

13. Ratcliffe, A., M. Doherty, R.N. Maini, and T.E. Hardingham. 1988. Increased levels of proteoglycan components in the synovial fluids of patients with acute joint disease. Ann. Rheum. Dis. 47:826-832.

14. Malik, S.C., J.P. Pearson, and C.W. Dick. 1984. A comparison of glycosaminoglycan content of human synovial fluid in different disease states. Biochem. Soc. Trans. 12:874.

15. Sandy, J.D., C.R. Flannery, P.J. Neame, and L.S. Lohmander. 1992. The structure of aggrecan fragments in human synovial fluid. Evidence for the involvement in osteoarthritis of a novel proteinase which cleaves the Glu 373-Ala 374 bond of the interglobular domain. J. Clin. Invest. 89:1512-1516.

16. Lohmander, L.S., P.J. Neame, and J.D. Sandy. 1993. The structure of aggrecan fragments in human synovial fluid: evidence that aggrecanase mediates cartilage degradation in inflammatory joint disease, joint injury, and osteoarthritis. Arthritis Rheum. 36:1214-1222.

17. Lewthwaite, J., S.M. Blake, T.E. Hardingham, P.J. Warden, and B. Henderson. 1994. The effect of recombinant human interleukin 1 receptor antagonist on the induction phase of antigen induced arthritis in the rabbit. $J$. Rheumatol. 21:467-472.

18. Arner, E.C. 1994. Effect of animal age and chronicity of interleukin-1 exposure on cartilage proteoglycan depletion in vivo. J. Orthop. Res. 12:321-330.

19. Cawston, T. 1993. Blocking cartilage destruction with metalloproteinase inhibitors: a valid therapeutic target. Ann. Rheum. Dis. 52:769-770.

20. Mort, J.S., G.R. Dodge, P.J. Roughley, J. Liu, S.J. Finch, G. DiPasquale, and A.R. Poole. 1993. Direct evidence for active metalloproteinases mediating matrix degradation in interleukin 1-stimulated human articular cartilage. $\mathrm{Ma}$ trix. 13:95-102.

21. Buttle, D.J., C.J. Handley, M.Z. Ilic, J. Saklatvala, M. Murata, and A.J. Barrett. 1993. Inhibition of cartilage proteoglycan release by a specific inactivator of cathepsin B and an inhibitor of matrix metalloproteinases: evidence for two converging pathways of chondrocyte-mediated proteoglycan degradation. Arthritis Rheum. 36:1709-1717.

22. Seed, M.P., S. Ismaiel, C.Y. Cheung, T.A. Thomson, C.R. Gardner, R.M. Atkins, and C.J. Elson. 1993. Inhibition of interleukin 1beta induced rat 
and human cartilage degradation in vitro by the metalloproteinase inhibitor U27391. Ann. Rheum. Dis. 52:37-43.

23. Hughes, C., B. Caterson, R.J. White, P.J. Roughley, and J.S. Mort. 1992. Monoclonal antibodies recognizing protease-generated neoepitopes from cartilage proteoglycan degradation. J. Biol. Chem. 267:16011-16014.

24. Hughes, C.E., B. Caterson, A.J. Fosang, P.J. Roughley, and J.S. Mort. 1995. Monoclonal antibodies that specifically recognise neoepitope sequences generated by "aggrecanase" and matrix metalloproteinase cleavage of aggrecan: application to catabolism in situ and in vitro. Biochem. J. 305:799-804

25. Lark, M.W., H. Williams, L.A. Hoerrner, J. Weidner, J.M. Ayala, C.F. Harper, A. Christen, J. Olszewski, Z. Konteatis, R. Webber et al. 1995. Quantification of a matrix metalloproteinase-generated aggrecan G1 fragment using monospecific anti-peptide serum. Biochem. J. 307:245-252.

26. Fosang, A.J., K. Last, P. Gardiner, D.C. Jackson, and L. Brown. 1995. Development of a cleavage site-specific monoclonal antibody for detecting metalloproteinase-derived aggrecan fragments: detection of fragments in human synovial fluids. Biochem. J. 310:337-343.

27. Singer, I.I., D.W. Kawka, E.K. Bayne, S.A. Donatelli, J.R. Weidner, H.R. Williams, J.M. Ayala, R.A. Mumford, M.W. Lark, T.T. Glant, et al. 1995. A metalloproteinase-generated neoepitope is induced and immunolocalizes in articular cartilage during inflammatory arthritis. J. Clin. Invest. 95:2178-2186.

28. Fosang, A.J., P.J. Neame, T.E. Hardingham, G. Murphy, and J.A. Hamilton. 1991. Cleavage of cartilage proteoglycan between G1 and G2 domains by stromelysins. J. Biol. Chem. 266:15579-15582.

29. Fosang, A.J., P.J. Neame, K. Last, T.E. Hardingham, G. Murphy, and J.A. Hamilton. 1992. The interglobular domain of cartilage aggrecan is cleaved by Pump, gelatinases and cathepsin B. J. Biol. Chem. 267:19470-19474.

30. Fosang, A.J., K. Last, V. Knäuper, P.J. Neame, G. Murphy, T.E. Hardingham, H. Tschesche, and J.A. Hamilton. 1993. Fibroblast and neutrophil collagenases cleave at two sites in the cartilage aggrecan interglobular domain. Biochem. J. 295:273-276.

31. Fosang, A.J., K. Last, V. Knäuper, G. Murphy, C.E. Hughes, and P.J. Neame. 1996. Degradation of cartilage aggrecan by collagenase-3 (MMP-13). FEBS Lett. 380:17-20.

32. Sandy, J.D., P.J. Neame, R.E. Boynton, and C.R. Flannery. 1991. Catabolism of aggrecan in cartilage explants. Identification of a major cleavage site within the interglobular domain. J. Biol. Chem. 266:8683-8685.

33. Ilic, M.Z., C.J. Handley, H.C. Robinson, and M.T. Mok. 1992. Mechanism of catabolism of aggrecan by articular cartilage. Archiv. Biochem. Biophys. 294:115-122.

34. Loulakis, P., A. Shrikhande, G. Davis, and C.A. Maniglia. 1992. N-Terminal sequence of proteoglycan fragments isolated from medium of interleukin-1-treated articular-cartilage cultures. Biochem. J. 284:589-593.

35. Ropes, M.W., G.A. Bennet, S. Cobb, R. Jacox, and R.A. Jessar. 1959. Revision of diagnostic criteria for rheumatoid arthritis. Ann. Rheum. Dis. 18: 49-53.

36. Altman, R., E. Asch, D. Bloch, G. Bole, D. Borenstein, K. Brandt, W. Christy, T.D. Cooke, R. Greenwald, M. Hochberh, et al. 1986. Development of criteria for the classification and reporting of osteoarthritis. Classification of osteoarthritis of the knee. Arthritis Rheum. 29:1039-1049.

37. Willkens, R.F., F.C. Arnett, and T. Bitter. 1982. Reiter's syndrome: evaluation of preliminary criteria for definite disease. Bull. Rheum. Dis 32:31-34.

38. Altman, D.G. 1991. Practical Statistics for Medical Research. Chapman \& Hall, London. 277-324.

39. Doege, K.J., M. Sasaki, T. Kimura, and Y. Yamada. 1991. Complete coding sequence and deduced primary structure of the human cartilage large aggregating proteoglycan, aggrecan. Human specific repeats and additional alternatively spliced forms. J. Biol. Chem. 266:894-902.

40. Farndale, R.W., C.A. Sayers, and A.J. Barrett. 1982. A direct spectrophotometric microassay for sulfated glycosaminoglycans in cartilage cultures. Connect. Tissue Res. 9:247-248.

41. Barry, F.P., L.C. Rosenberg, J.U. Gaw, T.J. Koob, and P.J. Neame. 1995. N- and O-linked keratan sulfate on the hyaluronan binding region of aggrecan from mature and immature bovine cartilage. J. Biol. Chem. 270:2051620524

42. Fairbanks, G., T.L. Steck, and D.F.H. Wallach. 1971. Electrophoretic analysis of the major polypeptides of the human erythrocyte membrane. Biochemistry. 10:2606-2616.

43. Mehmet, H., P. Scudder, P.W. Tang, E.F. Hounsell, B. Caterson, and T. Feizi. 1986. The antigenic determinant recognized by three monoclonal antibodies to keratan sulfate involve sulfated hepta or larger oligosaccharides of the poly (N-acetyllactosamine) series. Eur. J. Biochem. 157:385-391.

44. Caterson, B., A. Calabro, P.J. Donohue, and M.R. Jahnke. 1986. Monoclonal antibodies against cartilage proteoglycan and link protein. In Articular Cartilage Biochemistry. K.E. Kuettner, R. Schleyerbach, and V.C. Hascall, editors. Raven Press, New York. 59-73.

45. Dudhia, J., C.M. Davidson, T.M. Wells, D.H. Vynios, T.E. Hardingham, and M.T. Bayliss. 1996. Age-related changes in the content of the C-terminal region of aggrecan in human articular cartilage. Biochem. J. 313:933-940.

46. Hutton, S., J. Hayward, R.A. Maciewicz, and M. Bayliss. 1996. Agerelated and zonal distribution of aggrecanase activity in normal and osteoarthritic human articular cartilage. Trans. Orthop. Res. Soc. 21:150.

47. Heinegård, D., and I. Axelsson. 1977. Distribution of keratan sulfate in cartilage proteoglycans. J. Biol. Chem. 252:1971-1979.

48. Hanley, J.A., and A. Lippman-Hand. 1983. If nothing goes wrong is everything all right? Interpreting zero numerators. JAMA. 249:1743-1745.

49. Maniglia, C.A., P.P. Loulakis, A.V. Shrikhande, and G. Davis. 1991. IL-1 elevated PG degradation reveals NH2-terminal sequence homology. Trans. Orthop. Res. Soc. 16:193.

50. Plaas, A.H.K., and J.D. Sandy. 1993. A cartilage explant system for studies on aggrecan structure, biosynthesis and catabolism in discrete zones of the mammalian growth plate. Matrix. 13:135-147.

51. Bayne, E.K., S.A. Donatelli, I.I. Singer, J.R. Weidner, N.I. Hutchinson, L.A. Hoerrner, H.R. Williams, R.A. Mumford, L.S. Lohmander, and M.W. Lark. 1994. Detection of a metalloproteinase-generated aggrecan HABR fragment within human OA and RA cartilage. Trans. Orthop. Res. Soc. 19:308.

52. Fosang, A.J., K. Last, P.J. Neame, G. Murphy, V. Knäuper, H Tschesche, C.E. Hughes, B. Caterson, and T.E. Hardingham. 1994. Neutrophil collagenase (MMP-8) cleaves at the aggrecanase site E373-A374 in the interglobular domain of cartilage aggrecan. Biochem. J. 304:347-351.

53. Fosang, A.J., K. Last, P.J. Neame, T.E. Hardingham, G. Murphy, and J.A. Hamilton. 1993. Collagenase, plasmin and urokinase-type plasminogen ac tivator cleave the aggrecan interglobular domain. Orthop. Trans. 17:848-849.

54. Mok, M.T., M.Z. Ilic, C.J. Handley, and H.C. Robinson. 1992. Cleavage of proteoglycan aggregate by leucocyte elastase. Arch. Biochem. Biophys. 292: $442-447$.

55. Vilím, V., and A.J. Fosang. 1994. Proteoglycans isolated from dissociative extracts of differently-aged human articular cartilage: characterization of naturally-occuring hyaluronan-binding fragments of aggrecan. Biochem. J. 304: 887-894.

56. Singer, I.I., S. Scott, D.W. Kawka, E.K. Bayne, S.A. Donatelli, J.R. Weidner, H.R. Williams, R.A. Mumford, M.W. Lark, J. McDonnell, et al. 1995. Aggrecanase and metalloproteinase-specific aggrecan neoepitopes are induced in the articular cartilage of mice with collagen II arthritis. Trans. Orthop. Res. Soc. 20:330

57. Bayne, E.K., S.A. Donatelli, J. Sargeant, I.I. Singer, M.W. Lark, L.A Hoerrner, J.R. Weidner, H.R. Williams, R.A. Mumford, and L.S. Lohmander. 1995. Aggrecan fragments terminating in the sequence NITEGE373 are present within human OA and RA cartilage. Trans. Orthop. Res. Soc. 20:328.

58. Cawston, T.E., L. Weaver, R.J. Coughlan, M.V. Kyle, and B.L. Hazelman. 1989. Synovial fluids from infected joints contain active metalloproteinases and no inhibitory activity. Br. J. Rheumatol. 28:386-392.

59. Cawston, T., P. McLaughlan, R. Coughlan, V. Kyle, and B. Hazleman. 1990. Synovial fluids from infected joints contain metalloproteinase-tissue inhibitor of metalloproteinase (TIMP) complexes. Biochim. Biophys. Acta. 1033: 96-102.

60. Cole, A.A., and K.E. Kuettner. 1995. MMP-8 (neutrophil collagenase) mRNA and aggrecanase cleavage products are present in normal and osteoarthritic human articular cartilage. Acta Orthop. Scand. 66 (Suppl. 266):98-102.

61. Pratta, M.A., M.D. Tortorella, P. Gunyuzulu, G. Davis, H. George, and E.C. Arner. 1996. Co-induction of MMP-3 and MMP-8 synthesis in human chondrocytes in response to interleukin-1. Trans. Orthop. Res. Soc. 21:170.

62. Cole, A.A., S. Chubinskaya, B. Schumacher, K. Huch, G. Cs.-Szabó, J. Yao, K. Mikecz, K.A. Hasty, and K.E. Kuettner. 1996. Chondrocyte matrix metalloproteinase-8. Human articular chondrocytes express neutrophil collagenase. J. Biol. Chem. 271:11023-11026.

63. Lark, M.W., J.T. Gordy, J.R. Weidner, J. Ayala, J.H. Kimura, H.R. Williams, R.A. Mumford, C.R. Flannery, S.S. Carlson, M. Iwata, et al. 1995. Cellmediated catabolism of aggrecan. Evidence that cleavage at the "aggrecanase" site $\left(\mathrm{Glu}^{373}-\mathrm{Ala}^{374}\right)$ is a primary event in proteolysis of the interglobular domain. J. Biol. Chem. 270:2550-2556. 